\title{
Histopathological assessment of chronic Corynebacterium pseudotuberculosis infection in the reproductive tract and iliac lymph node of Katjang does
}

\begin{abstract}
Corynebacterium pseudotuberculosis is the etiological agent for caseous lymphadenitis (CLA), which is a chronic zoonotic disease that mostly infects sheep and goats worldwide. CLA causes significant financial losses in endemic countries due to decreased productivity and impaired reproduction in goats, sheep, and other small ruminants. In this study, 18 nonpregnant healthy Katjang does aged 2 years old were randomly divided into two groups of nine goats each. The first group were all experimentally inoculated with $1 \mathrm{ml}$ of $10^{7} \mathrm{cfu}$ of live $C$. pseudotuberculosis through intradermal route while the second group was given $1 \mathrm{ml}$ PBS (pH 7) intradermally. Three animals from each group were withdrawn and culled after 30, 60 and 90 days post infection and the reproductive organs (vagina, cervix, uterus, uterine horns, and ovaries) and iliac lymph nodes were collected in $10 \%$ buffered formalin, processed, sectioned, and stained with H\&E. Histopathological findings showed evidence of inflammation, degeneration, necrosis, and vascular changes in all the tissues examined. Inflammatory cell infiltration made up of neutrophils and macrophages were observed in all tissues. However, while neutrophil response was higher $(p<0.05)$ at the early phase (1 month) of the infection, macrophage response predominated at 3-months post infection. The distribution of necrosis and degeneration also increased $(p<0.05)$ between 1 and 3 months of infection in all tissues. Multifocal distribution of microabscesses indicative of pyogranuloma were observed in the cervix and iliac lymph nodes, with a higher $(p<0.05)$ distribution in the lymph nodes. Vascular congestion was also observed in all tissues, with varying severity of distribution at all sampling points. This study shows the time dependent effect of $C$. pseudotuberculosis infection on lesion severity in the reproductive system of goats, which necessitates the need for prompt diagnosis in order to prevent infertility and abortion in goat herds.
\end{abstract}

Keyword: Corynebacterium pseudotuberculosis; Histopathology; Reproductive organ; Chronic infection; Lymph node 